\title{
Calcium Spikes in Basal Dendrites of Layer 5 Pyramidal Neurons during Action Potential Bursts
}

\author{
Björn M. Kampa and Greg J. Stuart \\ Division of Neuroscience, John Curtin School of Medical Research, Australian National University, Canberra, Australian Capital Territory 0200, Australia
}

\begin{abstract}
Patch-clamp recording from dendrites has lead to a significant increase in our understanding of the mechanisms underlying signal integration and propagation in neurons. The majority of synaptic input to neurons, however, is made onto small-diameter dendrites, currently beyond the scope of patch-clamp recording techniques. Here we use both calcium and voltage imaging to investigate propagation of action potentials (APs) in fine basal dendrites of cortical layer 5 pyramidal neurons. High-frequency $(200 \mathrm{~Hz}) \mathrm{AP}$ bursts caused supralinear increases in dendritic calcium at distal, but not proximal, basal locations. Supralinear increases in dendritic calcium were also observed at distal basal locations during AP trains above a critical frequency $(\sim 100 \mathrm{~Hz})$. Using voltage imaging, we show that single APs undergo significant attenuation as they propagate into basal dendrites, whereas AP bursts lead to generation of dendritic calcium spikes. Focal and bath application of 4-AP increased the amplitude of calcium transients evoked by APs at distal, but not proximal, locations, suggesting that A-type potassium channels regulate AP backpropagation into basal dendrites. Finally, we show that pairing EPSPs with AP bursts is an effective means of activating synaptic NMDA receptors in basal dendrites. The experimental observations on the role of A-type potassium channels in regulation of AP backpropagation in basal dendrites, as well as the generation of dendritic calcium spikes during AP bursts, were reproduced in a morphologically realistic neuronal model with uniform distributions of dendritic sodium, calcium, and potassium channels. Together, these findings have important implications for understanding dendritic integration and synaptic plasticity in cortical basal dendrites.
\end{abstract}

Key words: action potential; dendrite; neocortex; backpropagation; STDP; imaging

\section{Introduction}

Information is represented and transferred in the CNS by fast voltage impulses called action potentials (APs). APs are initiated in the axon and propagate back into the dendritic tree of many neuronal types (Stuart et al., 1997). In cortical pyramidal neurons, this AP backpropagation is supported by dendritic voltagegated sodium channels, which enable somatic APs to actively propagate into the apical dendritic arbor in a decremental manner (Stuart and Sakmann, 1994). Most studies have focused on AP backpropagation into the larger-diameter apical dendrite of pyramidal neurons, because these dendrites are readily accessible to patch-clamp recordings. The majority of synaptic input to pyramidal neurons, however, is made onto the much finer basal dendrites (Larkman, 1991), currently beyond the scope of patchclamp recording techniques. Knowledge of AP backpropagation into these small dendrites is incomplete and will be particularly important during interaction of backpropagating APs with basal

Received July 24, 2005; revised May 26, 2006; accepted May 26, 2006.

This work was supported by funding from the Alexander von Humboldt Foundation and the Australian National Health and Medical Research Council. We thank Lucy Palmer for help with the voltage imaging and Fritjof Helmchen for help with the simulations.

Correspondence should be addressed to Dr. Greg J. Stuart, Division of Neuroscience, John Curtin School of Medical Research, Australian National University, Canberra, Australian Capital Territory 0200, Australia. E-mail: greg.stuart@anu.edu.au.

B. M. Kampa's present address: Department of Neurophysiology, Brain Research Institute, University of Zurich, CH-8057 Zurich, Switzerland. E-mail: kampa@hifo.unizh.ch.

DOI:10.1523/JNEUROSCI.3062-05.2006

Copyright $\odot 2006$ Society for Neuroscience $\quad$ 0270-6474/06/267424-09\$15.00/0 synaptic input. Basal dendrites of layer 5 pyramidal neurons receive the majority of synaptic input from neighboring pyramidal neurons (Markram et al., 1997b); synapses that have been extensively used to study spike-timing-dependent synaptic plasticity (STDP) (Markram et al., 1997a; Sjostrom et al., 2001, 2003). An understanding of AP backpropagation into basal dendrites is, therefore, critical to understanding the cellular mechanisms underlying STDP at these synapses.

Because of their inaccessibility to patch-clamp recording techniques, recent studies on information processing in basal dendrites of neocortical pyramidal neurons have used fluorescence imaging with calcium-sensitive dyes and voltage-sensitive dyes (VSDs) (Schiller et al., 2000; Antic, 2003; Milojkovic et al., 2004; Polsky et al., 2004). These studies indicate that synaptic inputs onto basal dendrites have the capacity to evoke NMDA spikes (Schiller et al., 2000) and plateau potentials (Milojkovic et al., 2004). In addition, they suggest that basal dendrites support robust AP backpropagation (Antic, 2003). Little is known, however, about the regulation of AP backpropagation during highfrequency AP bursts or the potential role of dendritic calcium electrogenesis in basal dendrites during burst firing. This issue is particularly important given the critical role of AP bursts in information processing (Lisman, 1997; Snider et al., 1998) and synaptic plasticity (Paulsen and Sejnowski, 2000).

Here we investigate the ability of single APs and highfrequency AP bursts to invade the basal dendrites of cortical layer 5 pyramidal neurons. In contrast to previous experimental and 
modeling studies (Vetter et al., 2001; Antic, 2003), we find that single backpropagating APs are severely attenuated in the distal basal dendrites of these neurons because of activation of A-type potassium channels. Importantly, APs can still depolarize the distal basal dendrites during high-frequency burst firing after activation of dendritic calcium channels and the generation of dendritic calcium spikes. These findings have important implications for understanding signal processing, as well as the cellular mechanisms underlying STDP, in basal dendrites

\section{Materials and Methods}

Wistar rats (3-4 weeks old) were anesthetized by inhalation of isoflurane and decapitated, and parasagittal brain slices $(300 \mu \mathrm{m})$ of somatosensory cortex were prepared according to guidelines approved by the Animal Ethics Committee of the Australian National University. During recording, slices were perfused with oxygenated extracellular solution [artificial CSF (ACSF)] containing the following (in $\mathrm{mm}$ ): $125 \mathrm{NaCl}, 3 \mathrm{KCl}, 1.25$ $\mathrm{NaH}_{2} \mathrm{PO}_{4}, 25 \mathrm{NaHCO}_{3}, 25$ glucose, $2 \mathrm{CaCl}_{2}$, and $1 \mathrm{MgCl}_{2}, \mathrm{pH} 7.4$ (with $5 \% \mathrm{CO}_{2}$ at $35 \pm 1{ }^{\circ} \mathrm{C}$ ). Whole-cell current-clamp recordings were made from the soma of layer 5 pyramidal neurons using a current-clamp amplifier [Molecular Devices (Palo Alto, CA) or Dagan (Minneapolis, MN)] with pipettes containing the following (in $\mathrm{mm}$ ): $135 \mathrm{~K}$-gluconate, $7 \mathrm{NaCl}$, 10 HEPES, $2 \mathrm{MgCl}_{2}$, and $2 \mathrm{Na}_{2} \mathrm{ATP}, \mathrm{pH} 7.2$ with $\mathrm{KOH}$. APs were elicited by brief somatic current injection ( $2 \mathrm{~ms}, 2-4 \mathrm{nA})$. Series resistance was corrected for with bridge balance and capacitance neutralization. Data were filtered at $10 \mathrm{kHz}$ and acquired at $20 \mathrm{kHz}$ on a Macintosh computer (Apple Computers, Cupertino, CA). AxoGraph software (Molecular Devices) was used for acquisition and analysis. Statistical significance was tested using the Student's $t$ test at a significance level of 5\%. Pooled data represents mean \pm SEM.

Calcium imaging. Changes in intracellular calcium concentration were imaged using a confocal microscope [LSM 510 (Zeiss, Oberkochen, Germany) or FV300 (Olympus Optical, Tokyo, Japan)] or a CCD camera (NeuroCCD-SM; Redshirt Imaging, Decatur, GA). Neurons were loaded via the recording pipette with the calcium-sensitive fluorescent dye Oregon Green BAPTA-1 (OGB-1) $\left(200 \mu \mathrm{M} ; K_{\mathrm{d}}\right.$ of $170 \mathrm{~nm}$; Invitrogen, Carlsbad, CA) or Oregon Green BAPTA-6F (OGB-6F) $\left(200 \mu \mathrm{M} ; K_{\mathrm{d}}\right.$ of $3 \mu \mathrm{M}$; Invitrogen) added to the pipette recording solution, and cells were filled for at least $30 \mathrm{~min}$ before recording. Calcium-sensitive dyes were excited using the 488 line of an argon laser (confocal microscope) or via a $475 \pm$ $15 \mathrm{~nm}$ bandpass filter (CCD camera), and emitted fluorescence was detected via a dichroic mirror $(510 \mathrm{~nm})$ and long-pass filter (505 or 510 $\mathrm{nm}$ ). Changes in fluorescence during APs and EPSPs were measured with line scans at $0.3-1 \mathrm{kHz}$ (confocal microscope) or from a small region of interest (ROI) taken at $1 \mathrm{kHz}$ frame rate (CCD camera) across basal dendritic shafts with spines. Calcium signals in spines and the adjacent dendrite were pooled. EPSP-evoked calcium transients were evoked by extracellular stimulation using a pipette filled with external solution plus Sulforhodamine 101 (Invitrogen) placed within $10 \mu \mathrm{m}$ of the imaged dendrite.

Imaging data were analyzed with Igor (WaveMetrics, Lake Oswego, OR) and expressed as the percentage change in fluorescence relative to baseline $(\Delta F / F)$ after background subtraction. For calculation of $\Delta F / F$ ratios during three APs relative to one $\mathrm{AP}$, data were only used if the peak of the one AP response was larger than two times the SD of the baseline noise. In experiments using drug applications, data points obtained during control and washout were averaged to correct for changes in baseline fluorescence during drug application. In the presence of 4-AP (4 mM), TTX was applied locally via a patch pipette filled with HEPES-buffered ACSF including both 4-AP (4 mM) and TTX $(2 \mu \mathrm{M})$. Pressure was applied to the pipette with a syringe, and data were collected during drug application. After release of pressure, TTX was washed out for up to $5 \mathrm{~min}$.

Voltage imaging. Neurons were filled with the voltage-sensitive dyes JPW1114 (Invitrogen) or its variant JPW3028 (synthesized by J. P. Wuskell and L. M. Loew, University of Connecticut, Farmington, CT) as described previously (Antic, 2003), except that a microscope halogen lamp was used as the excitation light source (Olympus Optical). As described by Antic (2003), cells were filled for $\sim 45 \mathrm{~min}$ and then repatched $\sim 2 \mathrm{~h}$ later with a pipette without dye. During this $2 \mathrm{~h}$ period, AP responses returned to close to control conditions (see Fig. 2 A). Precautions were taken to reduce photodamage. Only a small area $(\sim 30 \times 30 \mu \mathrm{m})$ was illuminated around the ROI $(3 \times 4.5 \mu \mathrm{m})$, which was visualized at high magnification $(60 \times$ objectives; Olympus Optical) as shown in Figure $2 \mathrm{~B}$. This increased spatial resolution and the signal-to-noise ratio and ensured that the ROI was in focus. As a consequence, different dendritic sites were imaged separately. Voltage-sensitive dyes were excited using a $520 \pm 45 \mathrm{~nm}$ bandpass filter, and emitted fluorescence was detected via a dichroic mirror $(575 \mathrm{~nm})$ and long-pass filter $(610 \mathrm{~nm})$. Data was obtained at $5 \mathrm{kHz}$ frame rate with a CCD camera (Redshirt Imaging) and digitally filtered with a Gaussian filter at $500 \mathrm{~Hz}$ after interpolation of data points to achieve a $20 \mathrm{kHz}$ sample rate. To allow comparison of imaged membrane voltage with that recorded intracellularly via the somatic recording pipette, somatic AP voltage recordings were acquired at the same frequency $(5 \mathrm{kHz})$ using the analog input of the camera software and were also filtered at $500 \mathrm{~Hz}$. AP latency was calculated based on the time difference between the peak of the recorded somatic AP and the dendritic AP-evoked fluorescence change divided by the distance from the middle of the soma to the dendritic location. The rise time of APevoked fluorescence transients was defined as the time taken to go from 10 to $90 \%$ of the peak amplitude. Traces are averages of 10-100 trials, and background illumination was subtracted. Data was stored as text files and analyzed with Igor (WaveMetrics).

Model of basal dendrites. Simulations of AP backpropagation in basal dendrites were performed using NEURON 5.6 (Hines and Carnevale, 1997) using a reconstructed layer 5 pyramidal neuron model adapted from Stuart and Spruston (1998). Intracellular resistivity, $R_{\mathrm{i}}$, was set to $150 \Omega . \mathrm{cm}$, membrane resistivity, $R_{\mathrm{m}}$, was set to $15,000 \Omega . \mathrm{cm}^{2}$, and membrane capacitance, $C_{\mathrm{m}}$, was set to $1 \mu \mathrm{F} / \mathrm{cm}^{2}$. The following active conductances were included: a fast-inactivating sodium and noninactivating potassium conductance were incorporated at a uniform density of $30 \mathrm{pS} / \mu \mathrm{m}^{2}$, except in the axon in which the sodium conductance density was raised to $30,000 \mathrm{pS} / \mu \mathrm{m}^{2}$. Throughout the basal dendrites, a fast-inactivating (A-type) potassium conductance was incorporated at a uniform density of $20 \mathrm{pS} / \mu \mathrm{m}^{2}$, together with a uniform distribution of a low-threshold (T-type) calcium conductance at a density of $3 \mathrm{pS} / \mu \mathrm{m}^{2}$ and a uniform distribution of a high-threshold calcium conductance at a density of $0.7 \mathrm{pS} / \mu \mathrm{m}^{2}$. All properties of active conductances were based on those used in a previous model by Schaefer et al. (2003). In addition, we included a calcium buffer and extrusion mechanism (Helmchen et al., 1996) with a buffer binding ratio of 20 and a clearance rate of $1200 \mathrm{~s}^{-1}$ as reported for small dendrites (Sabatini et al., 2002). The volume-averaged calcium concentration in each compartment was calculated based on the total calcium influx and the compartment volume. Simulations were performed at a nominal temperature of $35^{\circ} \mathrm{C}$.

\section{Results}

\section{Backpropagation of single APs versus bursts}

The basal dendrites of large layer 5 pyramidal neurons were imaged using a confocal microscope after being filled with fluorescent calcium-sensitive dyes and were identified by their origin at the soma, the presence of spines, and their typical radial branching pattern within layer 5 (Fig. $1 A$ ). APs were generated by somatic current injection and dendritic calcium influx associated with single APs and multiple AP bursts measured at different basal dendritic sites using the high-affinity dye OGB-1 (Fig. 1A). Single APs produced an increase in intracellular calcium that could be detected at all dendritic locations, with similar increases at proximal $(18.3 \pm 2.5 \% \Delta F / F$ peak; $<130 \mu \mathrm{m}$ from the soma; $n=12)$ and distal $(17.3 \pm 2.7 \% \Delta F / F$ peak; $>130 \mu \mathrm{m}$ from the soma; $n=18$ ) sites (Fig. $1 B, C)$. In contrast, high-frequency $(200$ $\mathrm{Hz}$ ) AP bursts generated significantly larger responses at distal dendritic locations (Fig. $1 B, C$ ). Similar responses were observed using the low-affinity dye OGB-6F $(n=10)$ (Fig. $1 D)$. Comparison of the response to a burst of three APs at $200 \mathrm{~Hz}$ relative to 
that evoked by a single AP indicated a distance-dependent, highly supralinear increase in calcium influx during AP bursts $(n=31)$ (Fig. $1 E)$. These experiments suggest that AP backpropagation into basal dendrites is significantly more effective during high-frequency bursts, as is the case in the distal apical dendrites of layer 5 pyramidal neurons (Larkum et al., 1999; Williams and Stuart, 2000).

Previous work indicates that dendritic calcium spikes are generated in the distal apical dendrites of layer 5 pyramidal neurons when the frequency of AP trains exceeds a critical value, usually $\sim 100 \mathrm{~Hz}$ (Larkum et al., 1999). This "critical frequency" is defined as the frequency of somatic AP firing in which the time integral of the recorded dendritic voltage becomes supralinear. An accompanying supralinear increase in intracellular calcium is observed at distal apical locations as a result of activation of dendritic voltage-activated calcium channels (Larkum et al., 1999). Similar findings were observed in distal basal dendrites, with a supralinear increase in dendritic calcium concentration at a similar critical frequency (Fig. 1F, G). Trains of five APs at various frequencies lead to a similar increase in calcium at proximal dendritic locations $(<130 \mu \mathrm{m}$ from the soma) (Fig. $1 G$ ). At distal sites in the basal dendrites $(>130 \mu \mathrm{m}$ from the soma), however, dendritic calcium influx was significantly larger during AP trains at frequencies above $100 \mathrm{~Hz}$ (Fig. $1 I-G$ ). On average, at distal dendritic locations ( $>130 \mu \mathrm{m}$ from the soma), calcium transients were fivefold $( \pm 1.2 ; n=4 ; p<0.01)$ larger during AP trains at supra-critical AP frequencies $(>100 \mathrm{~Hz})$ compared with the same number of APs evoked at $66 \mathrm{~Hz}$ (Fig. 1G). By analogy with previous findings in the apical dendrites of layer 5 pyramidal neurons, these results suggest that summation of APs in distal basal dendrites promotes activation of voltage-gated calcium channels, leading to the generation of calcium spikes.

\section{Voltage imaging of}

\section{dendritic electrogenesis}

In the absence of the ability to directly measure membrane potential, we used VSDs to investigate membrane potential changes in basal dendrites during AP bursts (Fig. $2 A-C$ ). A recent study using this method concluded that there is full propagation of single backpropagating APs throughout the entire basal dendritic tree (Antic, 2003). However, because it is not yet possible to quantify the magnitude of changes in membrane potential detected using VSDs, the absolute amplitude of the signals detected is unknown. What is possible is to compare the size of one signal relative to that of another detected at the same location. During backpropagation of AP bursts, we observed little change in the
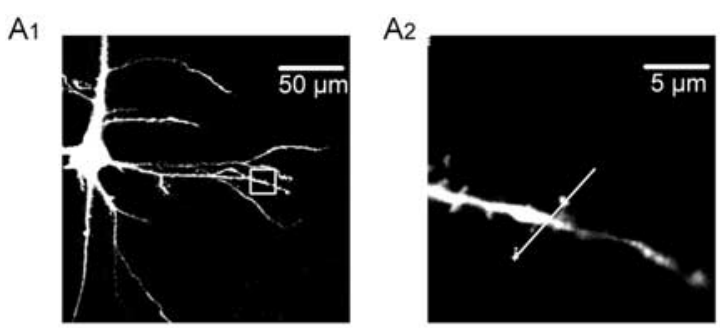

$\mathrm{A} 3$

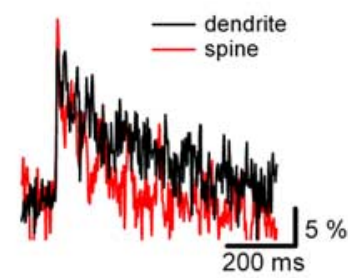

B
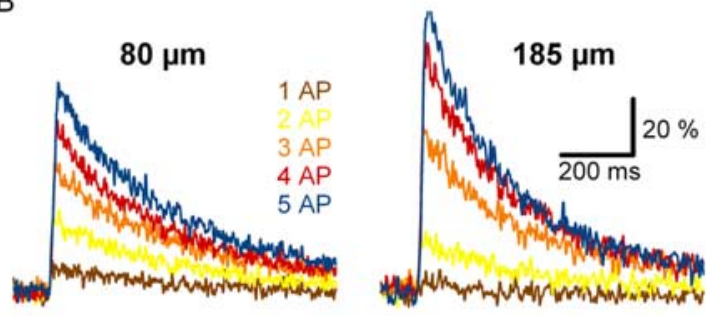

C

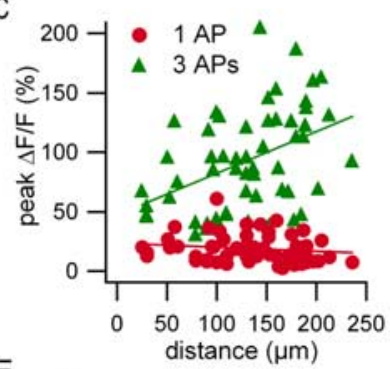

E

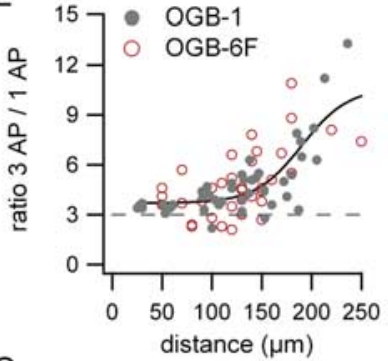

G
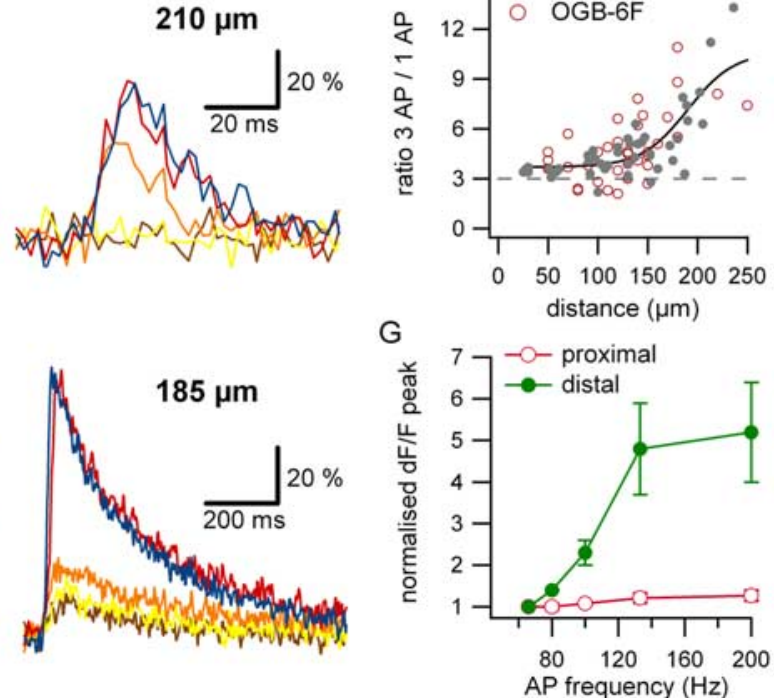

Figure 1. AP bursts evoke supralinear calcium rises in basal dendrites. A1, Confocal image of a layer 5 pyramidal neuron filled with 0GB-1. A2, Distal region of basal dendrite indicated by rectangle in $\boldsymbol{A 1}$. Line shows location of line scans. $\mathbf{A 3}$, Single APs evoke similar changes in intracellular calcium in the spine (red) and dendritic branch (black). $\boldsymbol{B}$, Changes in intracellular calcium in response to one to five APs (at $200 \mathrm{~Hz}$ ) in basal dendrites filled with OGB-1 at the indicated locations. C, Peak $\Delta F / F$ during one (red) or three (green) APs plotted against the distance of the recording site from the soma. Lines show linear fits to the data. D, Calcium imaging of the response to one to five $A P s$ (at $200 \mathrm{~Hz}$ ) in basal dendrites filled with $0 \mathrm{~GB}-6 \mathrm{~F}$ at the indicated locations. $\boldsymbol{E}$, Ratio of the response to three APs relative to one AP at different distances from the soma in experiments using 0GB-1 and 0GB-6F. Black line shows sigmoidal fit. Dashed line indicates a linear ratio. $F$, Calcium transients detected with 0 GB-1 at proximal and distal sites during trains of five APs at the indicated frequencies. G, Summary data of AP-evoked increases in calcium during trains of five APs at different frequencies. At a critical frequency above $100 \mathrm{~Hz}$, AP trains induced significantly more calcium influx in distal compared with proximal basal dendrites. Data are normalized to changes in calcium at an AP frequency of $66 \mathrm{~Hz}$.

amplitude or shape of the individual APs that makes up the burst at proximal basal sites $(<130 \mu \mathrm{m}$ from the soma) (Fig. $2 D$, gray traces). In contrast, at distal dendritic sites $(>130 \mu \mathrm{m})$, the amplitude of the third AP in a burst was significantly larger than that of the first (Fig. $2 D$, green traces), with the peak amplitude of the third AP in the burst being on average twofold greater than the first $(2.11 \pm 0.22 ; p<0.01)$ (Fig. $2 D$ ). Distal locations were imaged before proximal sites in approximately half of these experiments. The amplitude of the third AP relative to the first was not significantly different in these experiments compared with when proximal dendritic locations were imaged before distal sites (distal before proximal, mean of $2.22 \pm 0.29, n=8$; proximal 
A

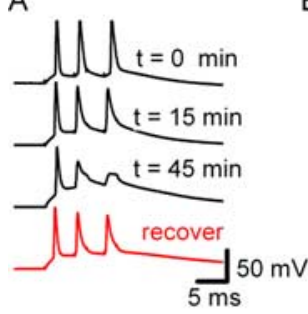

B

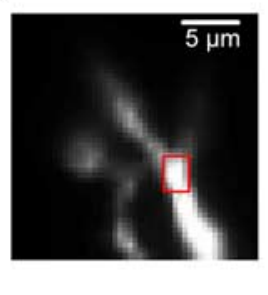

C

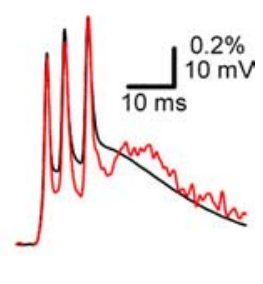

D
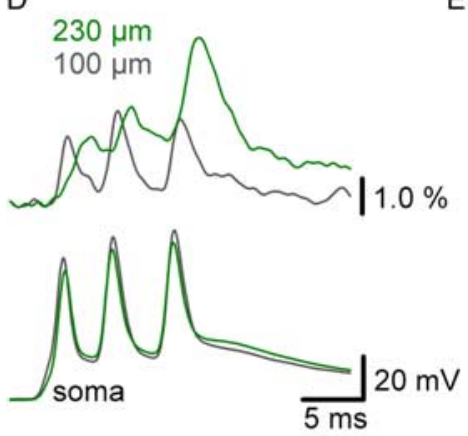

$E$
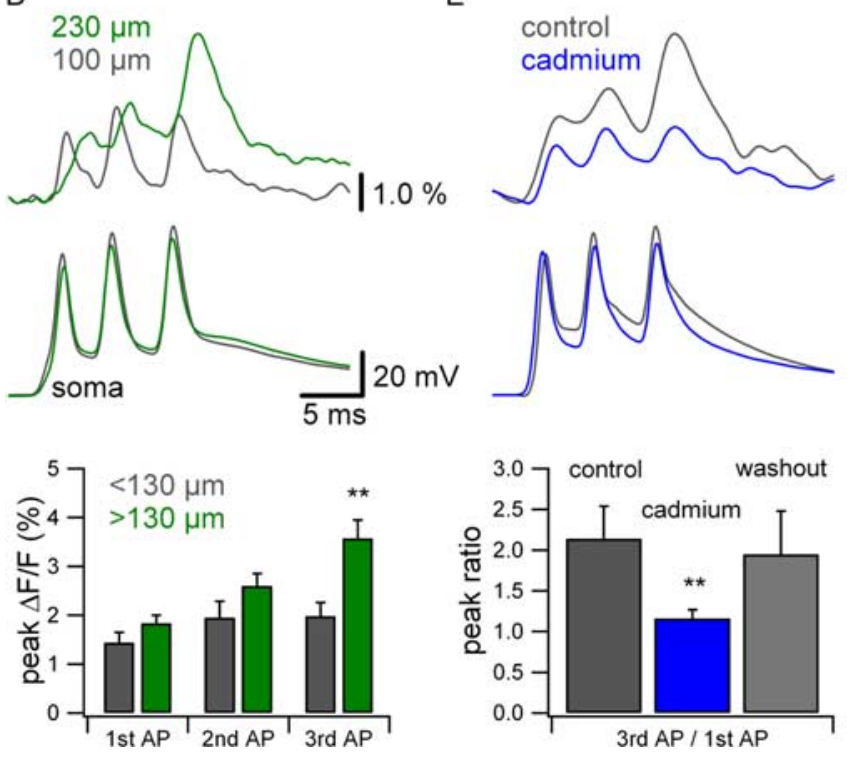

Figure 2. Voltage imaging of AP backpropagation in basal dendrites. $A, A$ three AP burst $(200 \mathrm{~Hz})$ recorded at the soma during filling (black traces) and after a $2 \mathrm{~h}$ incubation and recovery period (red trace). $\boldsymbol{B}$, Image of proximal dendrite (average of 30 trials). Rectangle indicates ROI. C, A three AP burst recorded at the soma (black) and imaged in proximal dendrite (red) shown in $\boldsymbol{B}$. Distance from soma was $30 \mu \mathrm{m}$. All data in $\mathbf{C}$ have been filtered at $500 \mathrm{~Hz}$. D, Fluorescence changes in distal (green; $230 \mu \mathrm{m}$ ) and proximal (gray; $100 \mu \mathrm{m}$ ) basal dendrites during a three AP burst $(200 \mathrm{~Hz})$. Bottom traces show simultaneously recorded somatic voltage. Traces from different distances were recorded separately. Bottom, Pooled peak $\Delta F / F$ for each AP in a three AP burst at proximal $(<130 \mu \mathrm{m} ; n=6)$ and distal $(>130 \mu \mathrm{m} ; n=12)$ basal dendritic sites. The peak of the third AP was significantly larger in distal dendrites. $p=0.005 . E$, A three AP burst as in $\boldsymbol{D}$ in control (gray) and cadmium ( $200 \mu \mathrm{m}$; blue) recorded $200 \mu \mathrm{m}$ from soma. Bottom, Average ratio of the third to the first AP peak in control $(n=6)$, cadmium $(n=$ $6)$, and after washout $(n=3) . p=0.047$.

before distal, mean of $1.83 \pm 0.21, n=6 ; p=0.29)$. Given that the region of illumination in these experiments is small $(\sim 30 \times$ $30 \mu \mathrm{m})$, these data rule out the possibility that photodamage at proximal basal sites is responsible for the observed reduction in amplitude of the third AP relative to the first at distal basal locations.

The most likely explanation of this finding is that, in contrast to the conclusions of Antic (2003), single APs undergo significant attenuation at distal basal dendritic locations, with the third AP in a high-frequency burst boosted as a result of recruitment of dendritic voltage-activated sodium or calcium channels. This explanation would be consistent with the observed supralinear increase in intracellular calcium at distal basal dendritic locations during AP bursts. To test for a role of dendritic calcium channels, we bath applied $200 \mu \mathrm{M}$ cadmium. Cadmium reduced the peak of the third AP relative to the first (Fig. $2 E$, top traces). On average, at distal basal locations ( $>130 \mu \mathrm{m}$ from the soma), the ratio of the third AP relative to the first was close to 1 in the presence of cadmium, and this ratio recovered to control levels after washout
(Fig. 2 E, bottom). These results provide direct evidence that single APs undergo significant attenuation as they backpropagate into distal basal dendrites, whereas AP bursts cause large depolarizations attributable to the generation of dendritic calcium spikes.

Additional evidence for attenuation of AP backpropagation in distal basal dendrites during single APs can be found by an examination of the properties of the first AP in the burst (Fig. 3A). The latency between the peak of somatic and dendritic APs was found to increase in a nonlinear manner with distance of the dendritic recording location from the soma (Fig. $3 B$ ). These data indicate that the conduction velocity of AP backpropagation into basal dendrites is not constant and is slower at more distal dendritic locations. The slowing of AP conduction velocity suggests that backpropagating APs undergo significant filtering as they propagating into basal dendrites. Consistent with this idea, the first AP in a burst showed a more than twofold increase in rise time (10$90 \%$ ) with recording distance from the soma that saturated at distal dendritic sites (Fig. 3C). These results are inconsistent with the idea that single APs propagate in a nondecremental manner into the distal basal dendrites. On the contrary, the observed slowing in AP velocity and increase in AP rise time is consistent with filtering of backpropagating APs by the passive cable properties of the distal basal dendrites. These data therefore support our conclusion that AP backpropagation into basal dendrites is decremental.

\section{Effect of A-type potassium channels}

One possible explanation for the attenuation of single backpropagating APs in distal basal dendrites is that this is attributable to activation of 4-AP-sensitive A-type potassium $\left(I_{\mathrm{A}}\right)$ channels, which have been shown previously to limit AP backpropagation in the apical dendrites of both hippocampal and cortical pyramidal neurons (Hoffman et al., 1997; Stuart and Hausser, 2001). To test this, we investigated the impact of 4-AP (4 mM) on APevoked dendritic calcium transients detected with the lowaffinity calcium-sensitive dye OGB-6F at proximal $(<130 \mu \mathrm{m}$ from the soma) and distal $(>130 \mu \mathrm{m})$ sites in the basal dendrites. To prevent epileptic discharges in the presence of 4-AP, DNQX $(10 \mu \mathrm{M})$ and APV $(50 \mu \mathrm{M})$ were added to the extracellular solution to block AMPA and NMDA receptors, and bicuculline (10 $\mu \mathrm{M})$ was added to block $\mathrm{GABA}_{\mathrm{A}}$ receptors. Single APs were evoked by a depolarizing/hyperpolarizing current sequence to block AP burst firing typically observed after bath application of 4-AP. At proximal sites, the calcium influx associated with backpropagation of single APs, as well as high-frequency AP bursts, was not changed after blockade of $I_{\mathrm{A}}$ channels (Fig. $4 \mathrm{~A}$ ). In contrast, bath application of 4-AP significantly enhanced the response to both single APs and AP bursts at distal basal locations $(p<0.05)$ (Fig. $4 B$ ). To investigate the role of D-type potassium channels, which would be expected to be blocked by high concentrations of 4-AP, we tested the effect of low concentrations of 4-AP $(100 \mu \mathrm{M})$. To increase the resolution of single AP responses, these experiments were performed using OGB-1. Bath application of low concentrations of 4-AP $(100 \mu \mathrm{M})$ had no significant effect on the single AP response at distal locations in basal dendrites $(>130 \mu \mathrm{m}$ from the soma; $n=4)$ (Fig. $4 C$ ). Together, these findings suggest that A-type, fast-inactivating, potassium channels in basal dendrites play an important role in limiting AP backpropagation, as has been observed previously in apical and oblique dendrites of pyramidal neurons (Hoffman et al., 1997; Stuart and Hausser, 2001; Frick et al., 2003). 
A

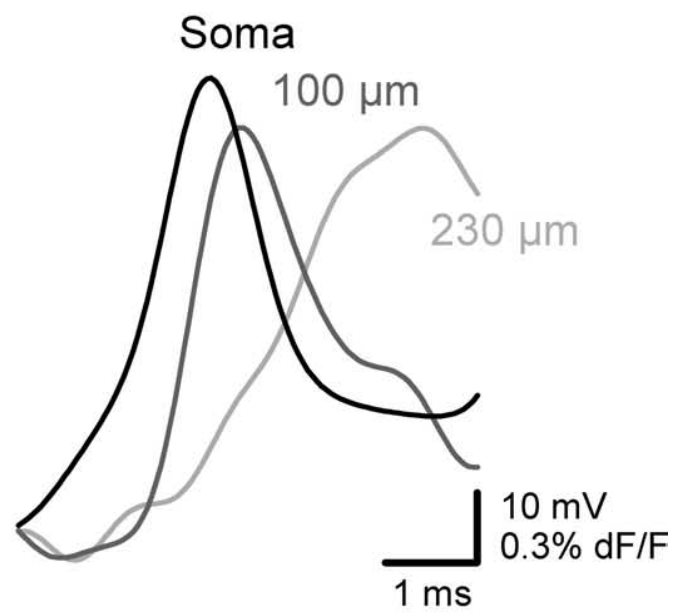

B

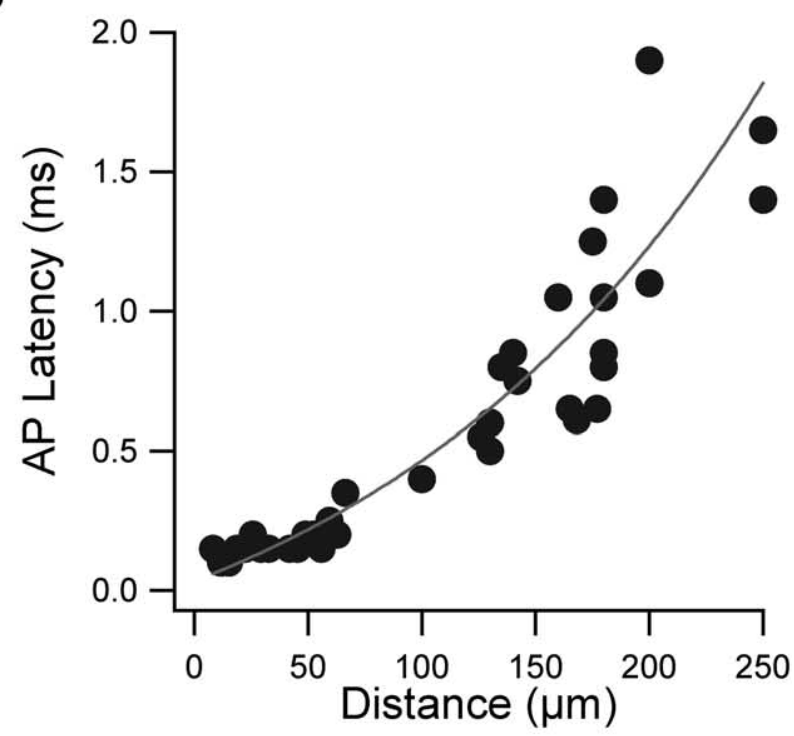

C

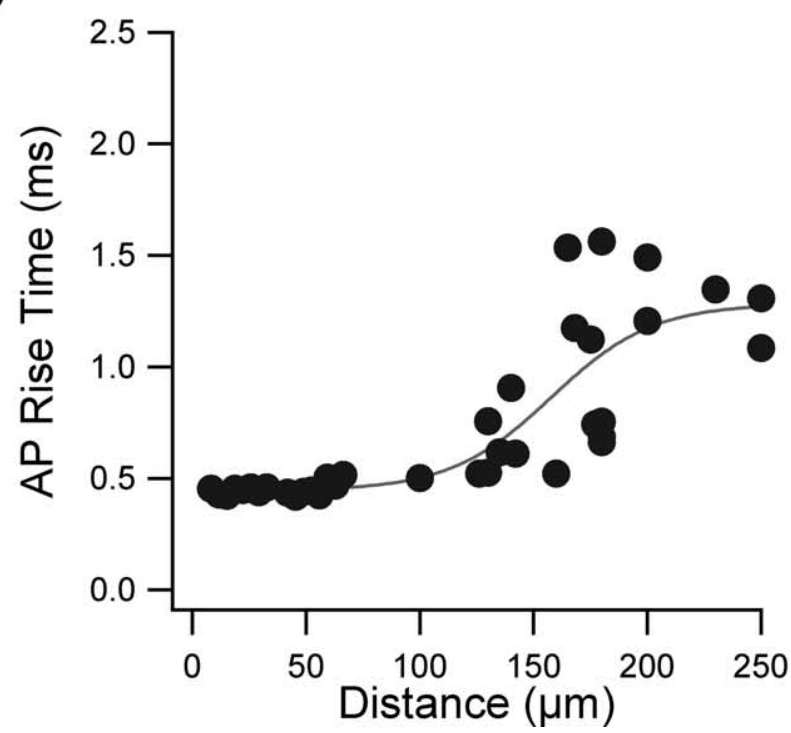

Figure 3. Properties of single backpropagating APs in basal dendrites. $\boldsymbol{A}$, Superimposed somatic voltage recording (black) and dendritic voltage imaging (gray traces). $\boldsymbol{B}$, AP peak latency increases in a nonlinear way with distance. Line shows exponential fit. $\boldsymbol{C}$, Dendritic AP rise time decreases with distance. Line shows sigmoidal fit.
A

proximal
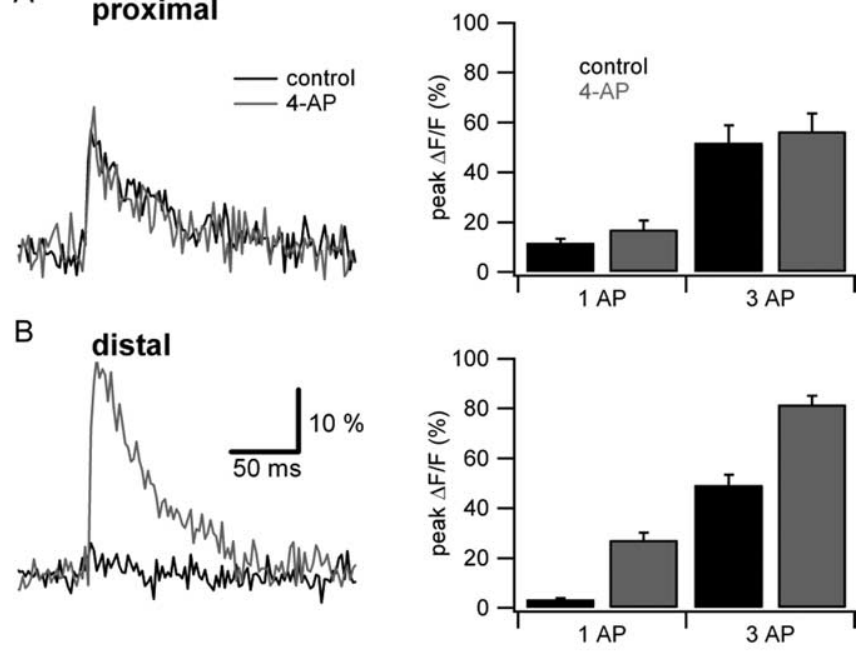

C
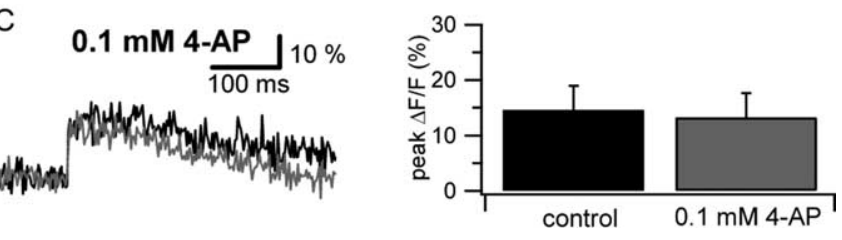

Figure 4. AP backpropagation in distal basal dendrites is modulated by A-type potassium channels. $A$, Calcium transients evoked by single backpropagating APs in control (gray) and after bath application of 4-AP (gray) at a proximal site ( $50 \mu \mathrm{m} ; A)$ and a distal dendritic site (204 $\mu \mathrm{m} ; \boldsymbol{B})$ of the same cell. Right bar graphs summarize changes in peak calcium transients evoked by one or three APs during 4-AP application $(4 \mathrm{~mm})$ for proximal $(<130 \mu \mathrm{m} ; n=6)$ and distal $(>130 \mu \mathrm{m} ; n=13)$ sites. Changes in intracellular calcium were imaged with OGB-6F. C, Impact of low $(100 \mu \mathrm{M})$ concentrations of 4-AP. Left, Calcium transients evoked by single backpropagating APs at distal basal locations in control (gray) and after bath application of 100 $\mu \mathrm{m}$ 4-AP (gray). Right, Pooled data of the average change in peak calcium transients evoked by single APs in control (black) and $100 \mu \mathrm{m}$ 4-AP (gray). Changes in intracellular calcium were imaged with OGB-1.

TTX sensitivity of AP backpropagation in distal basal dendrites

To investigate the role of dendritic sodium channels in AP backpropagation, we recorded AP-evoked changes in intracellular calcium during focal applications of TTX $(2 \mu \mathrm{M})$ in the presence or absence of bath applied 4-AP (Fig. 5A). Calcium responses detected with OGB-6F were sequentially imaged in distal basal (>150 $\mu \mathrm{m}$ from the soma) and proximal apical sites during focal application of TTX before, during, and after bath application of 4-AP. Local TTX applications in the absence of 4-AP had no significantly effect on the amplitude of AP-evoked calcium transients at distal basal dendritic sites (Fig. 5C) but significantly reduced calcium transients at proximal apical sites (Fig. 5C). In contrast, after blockage of $I_{\mathrm{A}}$ channels by bath application of 4-AP (4 mM), focal TTX applications to distal basal sites significantly reduced calcium transients evoked by single APs (Fig. 5B,C). These results show that, in the presence of 4-AP, backpropagating single APs recruit dendritic sodium channels, whereas this is not the case in the absence of 4-AP. These data suggest that, under control conditions, activation of dendritic $I_{\mathrm{A}}$ channels leads to failure of backpropagation of single APs in distal basal dendrites.

\section{Pairing EPSPs with AP bursts}

The results above suggest that AP bursts are likely to provide an effective stimulus for the relief of the voltage-dependent block of synaptic NMDA receptors by magnesium. To investigate this, we 
A

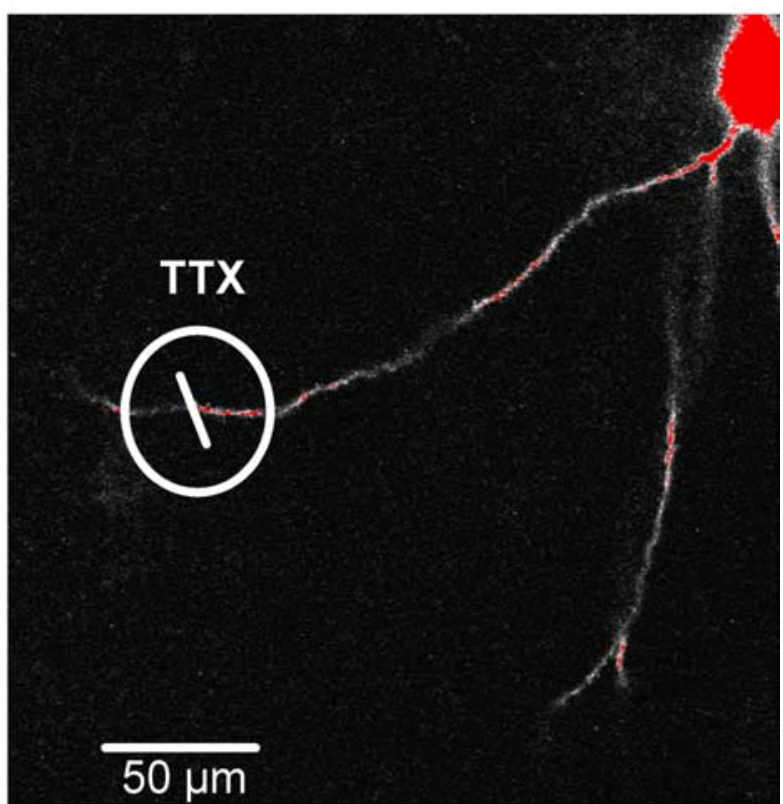

B

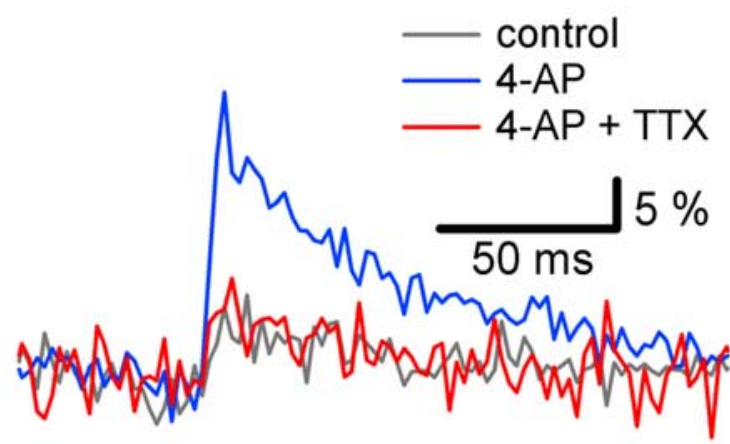

C

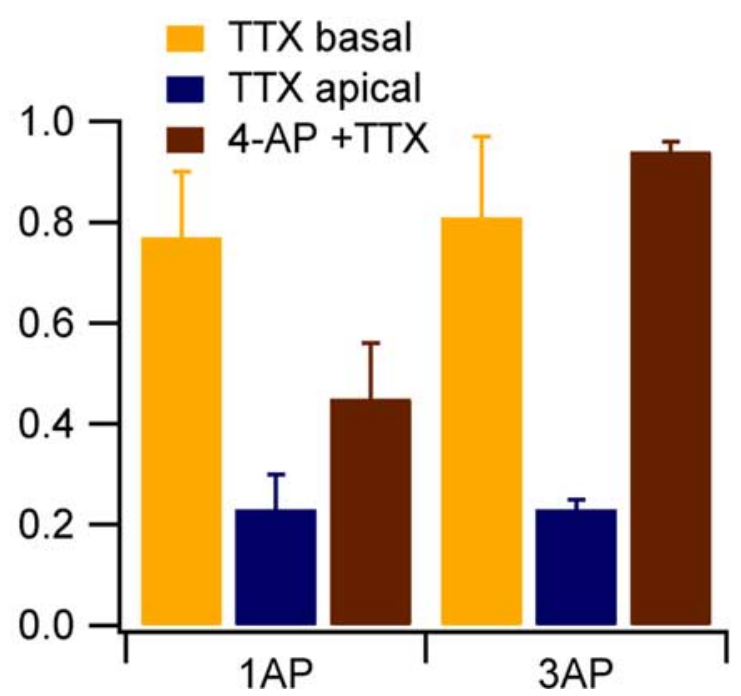

Figure 5. Activation of voltage-gated sodium channels in distal basal dendrites. $A$, Image of basal dendrite filled with OGB-6F. Line indicates location of confocal line scans, and circle shows location of focal TTX application. B, Calcium transients recorded at a distal basal site after generation of single APs in control (gray), in 4-AP (blue), and during focal application of TTX in the presence of 4-AP (red). C, Summary data showing the amplitude of AP-evoked calcium transients relative to control at distal basal locations during local application of TTX alone (yellow) and in the presence of 4-AP (brown; $n=6$ ) during single APs (left) and three AP bursts (right). The amplitude of AP-evoked calcium transients (relative to control) at proximal apical dendritic locations during local TTX applications is shown for comparison (blue).
A
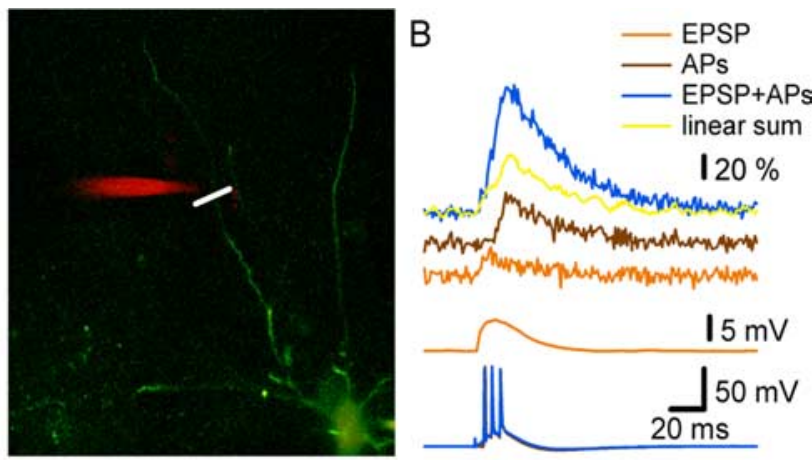

C

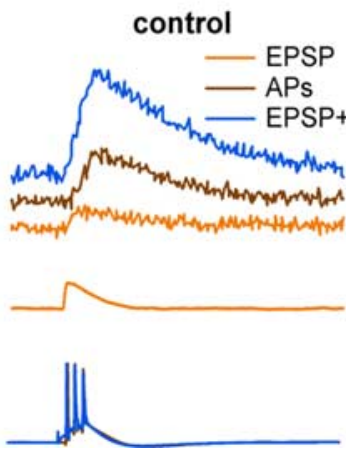

D

APV

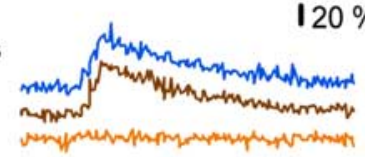

$15 \mathrm{mV}$

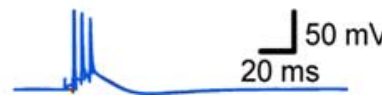

Figure 6. Calcium imaging pairing of AP bursts with EPSPs. A, Image of a layer 5 pyramidal neurons filled with $0 \mathrm{~GB}-6 \mathrm{~F}$ (soma at bottom right). The location of a line scan through a basal dendrite is indicated by the white bar. The extracellular stimulation pipette (red) is positioned in close proximity $(<10 \mu \mathrm{m})$ to the site in which calcium transients were recorded. $\boldsymbol{B}$, Dendritic calcium transients ( $\triangle F / F$; average of 3 trails) after extracellular stimulation of EPSPs alone (blue), three APs alone (red; $200 \mathrm{~Hz}$ ), or during pairing of EPSPs and AP bursts at $+10 \mathrm{~ms}$ (green; middle AP) imaged in a basal dendrite $175 \mu \mathrm{m}$ from the soma with OGB-6F. Somatic voltage during EPSP/AP pairing is also shown (black). Arrows indicate EPSP onset. Gray traces show the linear sum of the EPSP plus AP response. C, Increase in dendritic calcium $(\Delta F / F)$ after stimulation of EPSPs alone (blue), three APs alone (red), or during EPSP/AP pairing at $+10 \mathrm{~ms}$ (green) in control (top) or in the presence of APV ( $50 \mu \mathrm{m}$; bottom) imaged in a basal dendrite 80 $\mu \mathrm{m}$ from the soma. EPSP response and supralinear increase during pairing are blocked by APV $(50 \mu \mathrm{M})$.

used calcium imaging to detect changes in intracellular calcium during pairing of EPSP with AP bursts (Fig. 6A). These experiments indicated that pairing AP bursts (three APs at $200 \mathrm{~Hz}$ ) with EPSPs at positive times (+10 ms; middle AP) leads to supralinear increases in intracellular calcium (Fig. $6 B$ ), which were on average greater at distal dendritic locations (distal, $1.9 \pm 0.2$-fold, $n=$ $4,>150 \mu \mathrm{m}$ from the soma; proximal, $1.4 \pm 0.1$-fold, $n=4$, $<150 \mu \mathrm{m}$ from the soma). Supralinear increases in intracellular calcium during pairing of AP bursts with EPSPs were blocked by the NMDA receptor antagonist D-APV $(n=3)$, indicating the requirement of NMDA receptor activation (Fig. $6 C$ ). These observations show that appropriately timed AP bursts are effective at activating synaptic NMDA receptors in basal dendrites, suggesting a potential role for AP burst-evoked dendritic calcium spikes in STDP.

\section{Simulations of basal dendritic excitability}

Finally, we investigated whether the results above could be simulated using a morphologically realistic model of a layer 5 pyramidal neuron (Fig. 7A). Both low- and high-threshold voltageactivated calcium channels were included at a uniform density, because previous experimental studies suggested that they are present in the basal dendrites of layer 5 pyramidal neurons (Schiller et al., 1998). In addition, fast-inactivating A-type potas- 

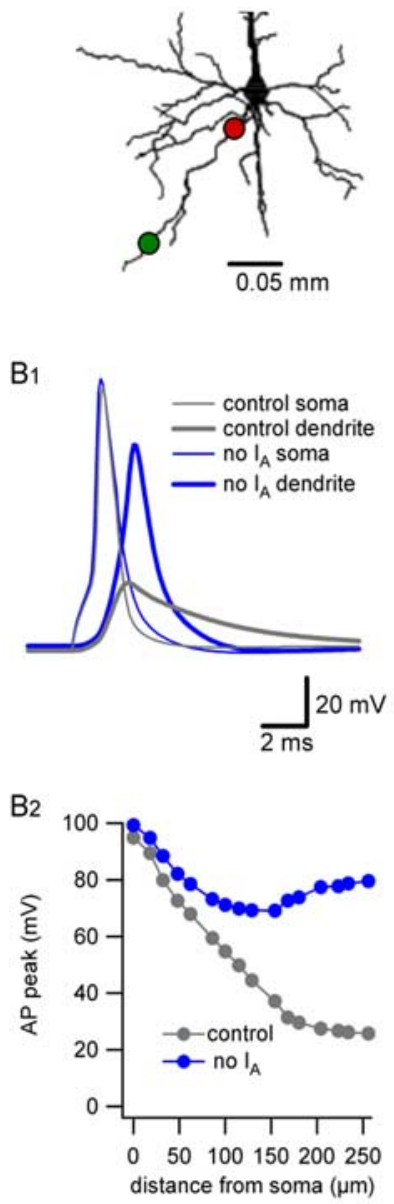

D1

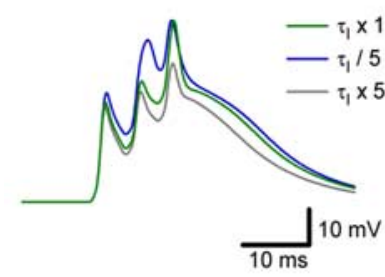

C1

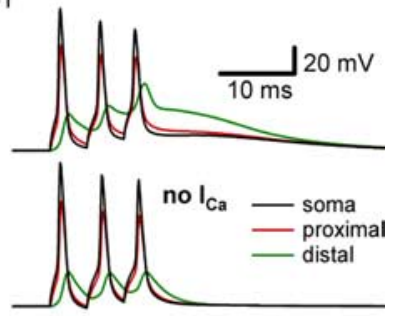

$\mathrm{C}_{2}$
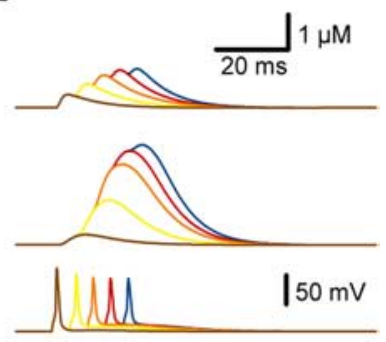

$\mathrm{C}_{3}$
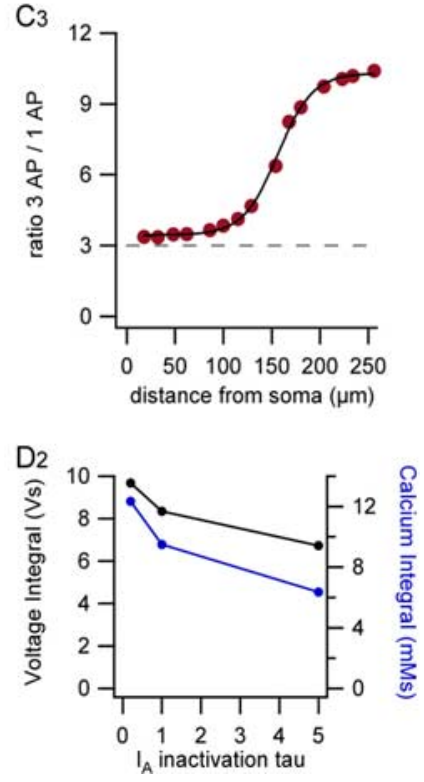

Figure 7. Simulation of AP backpropagation in basal dendrites in a model. $\boldsymbol{A}$, Image of compartmental model of a layer 5 pyramidal neuron showing the basal dendrites. Circles indicate a proximal (red; $48 \mu \mathrm{m}$ ) and distal (green; $204 \mu \mathrm{m}$ ) dendritic location. $\boldsymbol{B}$, A-type potassium currents $\left(I_{A}\right)$ reduce AP amplitude in distal basal dendrites. $B$ 1, Waveform of single AP at the soma (thin lines) and at a distal dendritic location (204 $\mu \mathrm{m}$ from soma; thick lines) in control (gray traces) and after removal of $I_{\mathrm{A}}$ channels (blue traces). B2, AP amplitude along a basal dendritic branch with (gray symbols) and without (blue symbols) $I_{\mathrm{A}}$. C, Calcium electrogenesis in basal dendrites. C1, Somatic (black) and dendritic AP waveforms (red, proximal; green, distal, as indicated in $\boldsymbol{A})$ during a three AP burst $(200 \mathrm{~Hz})$ under control conditions (top) and after removal of T-type and high-voltage-activated calcium channels from basal dendrites (bottom). Compare with Figure 2, D and E. C2, Intracellular calcium concentration at the basal dendritic locations indicated in $\boldsymbol{A}$ during trains of one to five APs ( $200 \mathrm{~Hz}$; color-coded traces). Somatic voltage is shown below. Compare with Figure 1D. C3, Ratio of the peak calcium transient evoked by three APs relative to one AP at different distances from the soma. Compare with Figure $1 E$. Black line shows sigmoidal fit. Dashed line indicates a linear ratio. D1, Dendritic AP waveforms during three AP bursts $(200 \mathrm{~Hz})$ in models with different inactivation time constants of $I_{A}$. Recorded $204 \mu \mathrm{m}$ from the soma. D2, Plot of voltage and calcium integral during AP bursts versus $I_{A}$ inactivation time constant (relative to control) for data like that in D1. sium channels were included at a uniform density throughout the basal dendrites. Single APs in this model failed to fully invade the distal region of basal dendrites, whereas removing $I_{\mathrm{A}}$ channels rescued AP backpropagation (Fig. $7 B$ ). Similar observations were made in models with a linear increase in the density of $I_{A}$, as in previous simulations of the role of A-type potassium channels in the regulation of AP backpropagation in hippocampal pyramidal neurons (Migliore et al., 1999). Consistent with our calcium imaging data, these simulations showed that AP bursts generated a dendritic calcium spike that was blocked when dendritic calcium channels were removed (Fig. 7C1). In addition, the intracellular calcium concentration at proximal basal sites increased linearly with the number of APs in a burst, whereas at distal basal sites, there was a highly supralinear increase during trains of three or more APs (Fig. 7C2). As a consequence, the ratio of the peak calcium transient evoked by three APs relative to that evoked by one AP showed a distance-dependent increase as observed experimentally (compare Figs. 7C3, 1E). These results were quantitatively similar to the experimental data obtained with calcium (Fig. $1 B-E$ ) and voltage (Fig. $2 D, E$ ) imaging.

We next investigated the role of fast inactivation of A-type potassium channels. Models in which the rate of $I_{\mathrm{A}}$ inactivation was slowed by a factor of 5 did not generate dendritic calcium spikes during three AP bursts, whereas speeding up the rate of inactivation by a factor of 5 generated calcium spikes after the second AP in a burst rather than the third (Fig. 7D1). These simulations show that the rate of inactivation of A-type potassium channels in basal dendrites plays a critical role in determining the extent of dendritic calcium electrogenesis during AP bursts (Fig. 7D2). Together, these findings indicate that the expression of fast-inactivating $I_{\mathrm{A}}$ channels on the one hand and low- and high-voltage-activated calcium channels on the other tune the distal basal dendrites to express dendritic calcium electrogenesis during AP bursts.

\section{Discussion}

The majority of synaptic input to cortical pyramidal neurons is made onto their basal dendrites (Larkman, 1991). An understanding of their electrophysiological properties is therefore essential to an understanding of how these neurons process and integrate synaptic input. In addition, the study of AP backpropagation into basal dendrites is particularly relevant to STDP. The experiments presented here demonstrate that single APs are significantly attenuated when invading the distal regions of basal dendrites, whereas AP bursts efficiently depolarize the distal basal dendrites as a result of activation of dendritic calcium channels and the generation of calcium spikes. Differences in the ability of single APs and AP bursts to invade distal basal dendrites were regulated by fast-inactivating, A-type potassium channels and may underlie the importance of AP burst firing for induction of synaptic plasticity (Paulsen and Sejnowski, 2000).

\section{Calcium imaging and electrogenesis in basal dendrites}

Previous work in apical dendrites of layer 5 pyramidal neurons indicates that single backpropagating APs evoke only small or negligible increases in intracellular calcium at distal locations, whereas high-frequency bursts lead to substantial distal calcium signals (Larkum et al., 1999). Similarly, in the basal dendrites of layer 5 pyramidal neurons, we found that the calcium signal associated with single backpropagating APs is small at distal sites, whereas the response to AP bursts increases supralinearly with distance of the recording site from the soma (Fig. 1). The observed supralinear increases in calcium are unlikely to be a consequence of 
the nonlinear binding properties of the calcium-sensitive dyes used, because similar supralinear increases were observed with high- and low-affinity dyes (Fig. 1E). A more likely explanation, which is consistent with data obtained in apical dendrites (Larkum et al., 1999), is that AP bursts cause regenerative activation of dendritic voltageactivated calcium channels, leading to generation of dendritic calcium spikes and substantial calcium influx.

\section{Voltage imaging of AP backpropagation in basal dendrites}

Optical methods to measure changes in ion concentrations or membrane voltage offer a great advantage over traditional recording methods when examining events in fine neuronal processes (Tsien, 1989; Denk et al., 1996). Although it is possible to convert fluorescence signals using ion-sensitive dyes into quantitative ion concentrations (assuming the affinity, binding kinetics, and dye concentration are known), it is much more difficult to calibrate signals using VSDs. Dye molecules have to be inserted into the cell membrane to sense the voltage across it. However, when introduced intracellularly, they also bind to the membranes of internal organelles that do not experience voltage changes during AP propagation, contributing to background fluorescence. Furthermore, perfect voltage control of basal dendrites is not possible via somatic recording (Spruston et al., 1993), making quantitative calibration of fluorescence changes at distal dendritic sites impossible. Therefore, only relative changes in dendritic voltage can be measured. In the case of full AP backpropagation, if no attenuation occurs, all APs in a train should have the same amplitude. This was found to be the case in the proximal basal dendrites. In distal basal dendrites, however, the peak of the third AP was much larger than that of the first as a result of generation of an AP burst-evoked dendritic calcium spike (Fig. 2 ). Given that previous voltage recordings of dendritic calcium spikes during AP bursts indicate that they peak at $\sim 0 \mathrm{mV}$ (Schiller et al., 1997; Larkum et al., 1999), the first AP in the burst is likely to be $\sim 30 \mathrm{mV}$ in amplitude, indicating significant attenuation. This conclusion is consistent with the slower rise time of backpropagating APs at distal basal locations (Fig. 3), the ability of 4-AP to increase the amplitude of distal but not proximal AP-evoked calcium transients (Fig. 4), and the finding that, in absence of 4-AP, local TTX applications did not affect the amplitude of distal dendritic calcium transients during single APs.

The conclusion that single APs attenuate as they propagate into distal basal dendrites is in contrast to that made by Antic (2003). One possible explanation for this difference is that the voltage-sensitive dye imaging in the study by Antic was performed at room temperature rather than physiological temperatures. Alternatively, the discrepancy between our study and that by Antic (2003) may simply be because we imaged more distal dendritic locations. Consistent with this idea, Antic (2003) also showed broadening of backpropagating APs at distal basal dendritic sites in a small subset of cells. It also seems possible that the main difference in the two studies is the conclusion drawn from the data rather than the actual data. No calibration was supplied to determine the amplitude of VSD signals observed in the study of Antic (2003). In our study, however, we were able to compare the relative amplitude of APs in a burst and found that they are not the same, as would be expected if APs propagate without amplitude attenuation.

\section{Mechanisms of AP backpropagation and calcium signals in basal dendrites}

In the hippocampus, CA1 neurons express $I_{\mathrm{A}}$ channels at high, non-uniform densities in their apical and oblique dendrites
(Hoffman et al., 1997; Frick et al., 2003). Activation of these channels causes a reduction in AP amplitude and the associated calcium signal (Hoffman et al., 1997; Frick et al., 2003). The apical dendrites of layer 5 pyramidal neurons also express $I_{\mathrm{A}}$ channels, albeit at a lower, uniform density (Korngreen and Sakmann, 2000; Bekkers, 2000). Activation of $I_{\mathrm{A}}$ channels contributes to failure of AP backpropagation in the apical dendrites of cortical layer 5 pyramidal neurons (Stuart and Hausser, 2001). Similarly, we show here that the AP-associated calcium influx during single APs in the distal basal dendrites of cortical layer 5 pyramidal neurons is reduced by the activation of $I_{\mathrm{A}}$ channels, because blockade of these potassium channels increased calcium influx triggered by backpropagating APs (Fig. 3). Furthermore, local TTX applications reduced calcium responses during APs when $I_{\mathrm{A}}$ channels were blocked but had no detectable affect under control conditions (Fig. 4). These data suggest that $I_{\mathrm{A}}$ channels contribute to attenuation and possible failure of AP backpropagation in distal basal dendrites.

In contrast, voltage imaging indicated that, at distal dendritic sites, AP bursts produced a large depolarization that was sensitive to blockers of voltage-activated calcium channels. This finding presumably underlies the supralinear calcium influx observed during AP bursts in distal basal dendrites. A likely scenario is that, at distal basal sites, attenuated and broadened backpropagating APs summate with each other when evoked at high frequencies. If the summed voltage reaches threshold for activation of dendritic calcium channels, their activation can become regenerative, leading to generation of a dendritic calcium spike. It has been reported previously that basal dendrites express a range of highvoltage-activated calcium channels, as well as nickel-sensitive low-threshold calcium channels (Schiller et al., 1998). Immunohistochemical studies reveal mainly P/Q-type channels in basal dendrites of layer 5 pyramidal neurons (Westenbroek et al., 1995), whereas N-type channels occur mainly on apical dendrites (Westenbroek et al., 1992) and L-type channels are restricted to the somatic and proximal dendritic region (Hell et al., 1993). Our simulations (Fig. 7) indicate that the expression of both lowthreshold T-type calcium channels and high-threshold calcium channels are required for the generation of dendritic calcium spikes in basal dendrites.

\section{Possible role of backpropagating APs in basal dendrites}

Backpropagating APs can act as a retrograde messenger informing the input to a neuron, the synapse, about the output of the neuron, the axonal AP. This may play an important role in STDP, in which the relative timing of the postsynaptic AP to the synaptic input determines whether the synapse undergoes potentiation or depression (Bi and Poo, 1998; Sjostrom et al., 2001). AP backpropagation is necessary for the induction of this kind of synaptic plasticity (Magee and Johnston, 1997). If single APs undergo significant attenuation, as appears to be the case in the distal basal dendrites, they will fail to deliver the depolarization needed to release the magnesium block inside the NMDA receptor channel pore. Dendritic calcium spikes evoked by AP bursts, conversely, have been shown to be very effective at removing the voltagedependent magnesium block of NMDA receptors (VargasCaballero and Robinson, 2003) and, as we show here, are effective triggers for NMDA receptor activation (Fig. 6). Together, these findings may explain why AP bursts are necessary for activation of synaptic NMDA receptors in basal dendrites (Kampa et al., 2006) and for the induction of synaptic plasticity in brain slices taken from mature ( $>3$-week-old) rats (Thomas et al., 1998; Pike et al., 1999; Kampa et al., 2006). Conversely, the finding that 
single APs at lower frequencies are sufficient for induction of STDP in younger (2-week-old) animals (Markram et al., 1997b; Sjostrom et al., 2001) suggests that backpropagation of single APs into basal dendrites is likely to be more robust at earlier stages of development.

Together, these data suggest that, during STDP, the extent of AP backpropagation and associated dendritic electrogenesis would be expected to play an important role in regulating the degree of synaptic NMDA receptor activation and so plasticity, a mechanism believed to be involved in learning and memory in the brain (Bliss and Collingridge, 1993).

\section{References}

Antic SD (2003) Action potentials in basal and oblique dendrites of rat neocortical pyramidal neurons. J Physiol (Lond) 550:35-50.

Bekkers JM (2000) Distribution and activation of voltage-gated potassium channels in cell-attached and outside-out patches from large layer 5 cortical pyramidal neurons of the rat. J Physiol (Lond) 525:611-620.

Bi G-Q, Poo M-M (1998) Synaptic modifications in cultured hippocampal neurons: dependence on spike timing, synaptic strength, and postsynaptic cell type. J Neurosci 18:10464-10472.

Bliss TV, Collingridge GL (1993) A synaptic model of memory: long-term potentiation in the hippocampus. Nature 361:31-39.

Denk W, Yuste R, Svoboda K, Tank DW (1996) Imaging calcium dynamics in dendritic spines. Curr Opin Neurobiol 6:372-378.

Frick A, Magee J, Koester HJ, Migliore M, Johnston D (2003) Normalization of $\mathrm{Ca}^{2+}$ signals by small oblique dendrites of CA1 pyramidal neurons. J Neurosci 23:3243-3250.

Hell JW, Westenbroek RE, Warner C, Ahlijanian MK, Prystay W, Gilbert MM, Snutch TP, Catterall WA (1993) Identification and differential subcellular localization of the neuronal class $C$ and class D L-type calcium channel alpha 1 subunits. J Cell Biol 123:949-962.

Helmchen F, Imoto K, Sakmann B (1996) $\mathrm{Ca}^{2+}$ buffering and action potential-evoked $\mathrm{Ca}^{2+}$ signaling in dendrites of pyramidal neurons. Biophys J 70:1069-1081.

Hines ML, Carnevale NT (1997) The NEURON simulation environment. Neural Comput 9:1179-1209.

Hoffman DA, Magee JC, Colbert CM, Johnston D (1997) $\mathrm{K}^{+}$channel regulation of signal propagation in dendrites of hippocampal pyramidal neurons. Nature 387:869-875.

Kampa B, Letzkus JJ, Stuart GJ (2006) Requirement of dendritic calcium spikes for induction of spike-timing dependent synaptic plasticity. J Physiol (Lond) 574.1:283-290.

Korngreen A, Sakmann B (2000) Voltage-gated K+ channels in layer 5 neocortical pyramidal neurones from young rats: subtypes and gradients. J Physiol (Lond) 525:621-639.

Larkman AU (1991) Dendritic morphology of pyramidal neurones of the visual cortex of the rat. III. Spine distributions. J Comp Neurol 306:332-343.

Larkum ME, Kaiser KM, Sakmann B (1999) Calcium electrogenesis in distal apical dendrites of layer 5 pyramidal cells at a critical frequency of backpropagating action potentials. Proc Natl Acad Sci USA 96:14600-14604.

Lisman JE (1997) Bursts as a unit of neural information: making unreliable synapses reliable. Trends Neurosci 20:38-43.

Magee JC, Johnston D (1997) A synaptically controlled, associative signal for Hebbian plasticity in hippocampal neurons. Science 275:209-213.

Markram H, Lübke J, Frotscher M, Sakmann B (1997a) Regulation of synaptic efficacy by coincidence of postsynaptic APs and EPSPs. Science 275:213-215.

Markram H, Lubke J, Frotscher M, Roth A, Sakmann B (1997b) Physiology and anatomy of synaptic connections between thick tufted pyramidal neurones in the developing rat neocortex. J Physiol (Lond) 500:409-440.

Migliore M, Hoffman DA, Magee JC, Johnston D (1999) Role of an A-type $\mathrm{K}^{+}$conductance in the back-propagation of action potentials in the dendrites of hippocampal pyramidal neurons. J Comput Neurosci 7:5-15.
Milojkovic BA, Radojicic MS, Goldman-Rakic PS, Antic SD (2004) Burst generation in rat pyramidal neurones by regenerative potentials elicited in a restricted part of the basilar dendritic tree. J Physiol (Lond) 558:193-211.

Paulsen O, Sejnowski TJ (2000) Natural patterns of activity and long-term synaptic plasticity. Curr Opin Neurobiol 10:172-179.

Pike FG, Meredith RM, Olding AW, Paulsen O (1999) Postsynaptic bursting is essential for "Hebbian" induction of associative long-term potentiation at excitatory synapses in rat hippocampus. J Physiol (Lond) 518:571-576.

Polsky A, Mel BW, Schiller J (2004) Computational subunits in thin dendrites of pyramidal cells. Nat Neurosci 7:621-627.

Sabatini BL, Oertner TG, Svoboda K (2002) The life cycle of $\mathrm{Ca}^{2+}$ ions in dendritic spines. Neuron 33:439-452.

Schaefer AT, Larkum ME, Sakmann B, Roth A (2003) Coincidence detection in pyramidal neurons is tuned by their dendritic branching pattern. J Neurophysiol 89:3143-3154.

Schiller J, Schiller Y, Stuart G, Sakmann B (1997) Calcium action potentials restricted to distal apical dendrites of rat neocortical pyramidal neurons. J Physiol (Lond) 505:605-616.

Schiller J, Schiller Y, Clapham DE (1998) NMDA receptors amplify calcium influx into dendritic spines during associative pre- and postsynaptic activation. Nat Neurosci 1:114-118.

Schiller J, Major G, Koester HJ, Schiller Y (2000) NMDA spikes in basal dendrites of cortical pyramidal neurons. Nature 404:285-289.

Sjostrom PJ, Turrigiano GG, Nelson SB (2001) Rate, timing, and cooperativity jointly determine cortical synaptic plasticity. Neuron 32:1149-1164.

Sjostrom PJ, Turrigiano GG, Nelson SB (2003) Neocortical LTD via coincident activation of presynaptic NMDA and cannabinoid receptors. Neuron 39:641-654.

Snider RK, Kabara JF, Roig BR, Bonds AB (1998) Burst firing and modulation of functional connectivity in cat striate cortex. J Neurophysiol $80: 730-744$.

Spruston N, Jaffe DB, Williams SH, Johnston D (1993) Voltage- and spaceclamp errors associated with the measurement of electrotonically remote synaptic events. J Neurophysiol 70:781-802.

Stuart G, Spruston N (1998) Determinants of voltage attenuation in neocortical pyramidal neuron dendrites. J Neurosci 18:3501-3510.

Stuart G, Spruston N, Sakmann B, Häusser M (1997) Action potential initiation and backpropagation in neurons of the mammalian central nervous system. Trends Neurosci 20:125-131.

Stuart GJ, Hausser M (2001) Dendritic coincidence detection of EPSPs and action potentials. Nat Neurosci 4:63-71.

Stuart GJ, Sakmann B (1994) Active propagation of somatic action potentials into neocortical pyramidal cell dendrites. Nature 367:69-72.

Thomas MJ, Watabe AM, Moody TD, Makhinson M, O’Dell TJ (1998) Postsynaptic complex spike bursting enables the induction of LTP by theta frequency synaptic stimulation. J Neurosci 18:7118-7126.

Tsien RY (1989) Fluorescent probes of cell signaling. Annu Rev Neurosci 12:227-253.

Vargas-Caballero M, Robinson HP (2003) A slow fraction of $\mathrm{Mg}^{2+}$ unblock of NMDA receptors limits their contribution to spike generation in cortical pyramidal neurons. J Neurophysiol 89:2778-2783.

Vetter P, Roth A, Hausser M (2001) Propagation of action potentials in dendrites depends on dendritic morphology. J Neurophysiol 85:926-937.

Westenbroek RE, Hell JW, Warner C, Dubel SJ, Snutch TP, Catterall WA (1992) Biochemical properties and subcellular distribution of an N-type calcium channel alpha 1 subunit. Neuron 9:1099-1115.

Westenbroek RE, Sakurai T, Elliott EM, Hell JW, Starr TV, Snutch TP, Catterall WA (1995) Immunochemical identification and subcellular distribution of the $\alpha 1 \mathrm{~A}$ subunits of brain calcium channels. J Neurosci 15:6403-6418.

Williams SR, Stuart GJ (2000) Backpropagation of physiological spike trains in neocortical pyramidal neurons: implications for temporal coding in dendrites. J Neurosci 20:8238-8246. 